\title{
Supporting Cross-Organizational Assimilation of IoT Innovation Exemplified by the ChainPORT Initiative
}

\author{
Jöran Tesse \\ University of Hamburg \\ tesse@informatik.uni-hamburg.de \\ Sebastian Saxe \\ Hamburg Port Authority \\ sebastian.saxe@hpa.hamburg.de
}

\begin{abstract}
The chainPORT community of port authorities (PAs) around the world gave their commitment to collaborate. Many PAs developed Internet of Things (IoT) based solutions to increase their operational efficiency. Within its IT solutions workgroup, the challenge of supporting the diffusion and assimilation of these IoT innovations was adressed by creating a centralized communication platform for IoT solutions to allow inter-organizational knowledge exchange. We draw upon the knowledge gained by analyzing 24 solutions from 8 port authorities and present concepts on how the specific challenges in this setting were adressed and what principles guided the creation of the emerging IT artifact.
\end{abstract}

\section{Introduction}

Organizational competitiveness has been widely accepted to be strongly influenced by an organization's ability to innovate [1]. While innovativeness can be achieved through invention, another important source of innovation is the assimilation of existing innovations to an organization's specific situation. Innovations can be seen to follow general trends, like the Internet of Things (IoT) which is anticipated and observed to impact many different industries [2]. Within this stream of innovations, a multitude of challenges arises, both from a technological perspective, as the increasing numbers of interconnected devices comes with increased technological complexity and heterogeneity, and from a business perspective, where the link to the physical world impacts especially the strategic level [3].

The logistics industry started adopting smart technologies relatively early [4] and, especially in the maritime logistics industry, continuous efforts have been undertaken to increase efficiency through innovation [5].

\author{
Ingrid Schirmer \\ University of Hamburg \\ schirmer@informatik.uni-hamburg.de \\ Ulrich Baldauf \\ Hamburg Port Authority \\ ulrich.baldauf@hpa.hamburg.de
}

Nonetheless, digitization is still at an early stage, as some areas were observed to be widely untouched by smart technology not too long ago [6].

At the port of Hamburg, digitization played an important role in recent years, due to the need to increase efficiency. The port of Hamburg is located at the center of the city of Hamburg and has very limited potential of spatial growth. Therefore, the increasing amounts of freight [7] have to be handled by utilizing the available area more efficiently. To increase efficiency through digitization, the Hamburg Port Authority presented their smartPORT-initiative's results when hosting the 2015 IAPH international port conference. After a consolidation phase of their smart technology projects [8], they can currently be seen as a driving force in the relatively young chainPORT initiative, a global community of port authorities (PAs) committed to collaborate.

The field of innovation diffusion research is concerned with the process of an innovation spreading through a social system, spanning from knowledge of an innovation to, potentially, its adoption and implementation [9]. This field of research as well as the research field of enterprise architecture management have been identified to be able to support the diffusion of Internet of Things innovations throughout the chainPORT community [10], although only a vague definition from a broader diffusion perspective has been stated.

In contrast to the diffusion of innovations, the innovation assimilation process focuses on how an innovation is adopted by an organization and how it is adjusted to accommodate the organization's specific context. The question to be answered here is how the inter-organizational assimilation of Internet of Things innovation can be supported. 


\section{Context}

The presented research was conducted at the port of Hamburg in close cooperation with the local port authority. Organizationally, the research was situated within the solutions workgroup of the chainPORT initiative. Previous research projects with the Hamburg Port Authority have created a solid basis of trust and transparency between the university of Hamburg and the practitioner, which led to the opportunity to be included in the solutions workgroup's tasks.

Port authorities are commonly responsible for developing, providing and maintaining traffic infrastructure for road traffic, waterways and railways. Especially their role as a coordinator across different types of traffic infrastructure can be highly complex, for example when coordinating the arrival of large container ships with tidal flows, movable bridges, trucks coming to pick up the containers, and the few parking lots inside and in front of the port area.

\subsection{Hamburg Port Authority}

Hamburg's local port authority faces the challenge of having to handle increasing amounts of freight on an area that can not be expanded, as the port is surrounded by the city of Hamburg. Previously, the HPA tackled this problem with their smartPORT initiative, aimed to increase organizational efficiency. Several of these projects were showcased at the 2015 IAPH international port conference that was hosted by Hamburg. After a consolidation phase, accompanied by research projects with the University of Hamburg, the chainPORT initiative was formed with the HPA as a founding member. The goal of this new initiative was less an internal perspective focussing on self-optimization, but rather a global perspective perspective to increase efficiency in the business ecosystem as a whole.

\section{2. smartPORT Initiative}

Initially, more than 20 exploratory projects were launched within the smartPORT initiative, ranging from process optimization projects to technology evaluation projects. Currently, the efforts are still ongoing and advertise over-arching digitization efforts at the Hamburg Port Authority. Many of the early projects are operational and have proven to deliver value. Following the 2015 international port conference, the University of Hamburg accompanied the HPA's consolidation phase that followed their initial exploratory projects $[8,11]$

\section{3. chainPORT Initiative}

The group of ports within the chainPORT network is a small but global group of ports around the globe, and most of the participating ports are the largest port of their respective country. Although these ports are competitors with similar goals and share many customers, the goal is to collaborate and to move "beyond bilateral partnerships" to face "changing competitive challenges" [12]. Participating members are (west to east): Los Angeles (USA), Montreal (Canada), Barcelona (Spain), Antwerp (Belgium), Rotterdam (Netherlands), Hamburg (Germany), Busan (South Korea) and Singapore (Singapore).

\subsection{IT Solutions Workgroup}

The IT solutions workgroup tries to capture knowledge about IT solutions of different stages (idea, piloted, productive). One first goal of this workgroup was to create a common understanding of what an IT solution is, as this relatively common term has not been defined from an architectural and IS management perspective [10].

\subsection{ChainPORT Projects}

We introduce the 24 IT solutions that were examined and modelled during our research. Due to the context of maritime logistics, the majority of solutions can be seen to be IoT related.

Digital 3D Port Model (1): Step-by-step creation of a full digital copy of the port area to gain experience in merging various sources of 3d-data, for example the incorporation of the current state of a building during its construction into a virtual reality (display) system. The solution is piloting for planning activities.

Port Monitor (2): Incorporation of various data sources to create a holistic view of the current state of the port, most importantly the traffic situation and infrastructure status on the different traffic carriers. This system is in full productive usage.

Smart Railway Switch (3): Implementation of new sensors to allow predictive maintenance for railroad switched, thus reducing the need to send maintenance teams on a fixed schedule while reducing the risk of unexpected failure. This solution is productive but not fully rolled out due to pending regulatory changes.

Sharing Port Information (4): Project proposal to gain experience in connecting data streams between ports. This solution is still in ideation state.

Smart Sounding Table (5): Digitization of vessel movement planning, utilizing a touch screen and various data sources such as vessel information 
systems, river depth measurements/forecasts and movable infrastructure information. The solution is used productively.

Road Traffic Simulation System (6): Explores advantages of incorporating new sensor types into complex road traffic simulations, allowing more accurate traffic estimations and traffic forecasts. The solution is operational but results are partially merged with legacy system data.

Virtual Gates (7): Assessing gates' traffic situations by sharing real-time traffic information between terminal operators and port authority. This solution is used productively.

Port Links (8): Collaborative platform to allow estimation of total $\mathrm{CO}_{2}$ emission on complex routes. The solution is operational but not fully rolled out.

Internet Infrastructure (9-11): Four ports reported infrastructure projects to provide wireless internet publicly within the port area or to carry out an internal network redesign, though the employed technologies vary (public: 4G, WiFi; internal: optical fiber, WiFi). They are seen as a prerequisite for internet-based services as well as new Sensors (IoT-specific challenges).

Cyber Security Center (12, 13): Two ports addressed possible cyber security threats by establishing operational cyber security groups. These develop both technological resilience as well as raising awareness for phishing and social engineering, and establishing counter measures. These solutions are operational.

Single Window Projects $(14,15)$ : Two ports each reported a project aimed at creating a single front-end for several customer-facing services to streamline the customer journey and increase the incorporated services' usability. The services are related to berths, customs and other port authority tasks. Both solutions are in productive usage.

Data Analytics Dashboard (16): Creating a dashboard service aimed at creating a holistic view by merging reporting visualizations of multiple stakeholder groups. The scope also includes reporting process digitization, as these reports were previously created and sent manually. This solution is operational and has further development potential.

Surveillance Drones (17): Project aimed at leveraging small unmanned aerial vehicles to lower response times in cases of disasters like oil spills. The solution is in an ongoing pilot state.

Push Communication (18): Mobile app aimed at allowing provider-to-consumer communication to notify about certain events rather then requiring customers to actively check for events. This solution is used productively.
Trucking Portal (19): Sensor project aimed at more accurately capturing truck turn times. The data is used in several services and publicly accessible in a web portal for truckers. This solution is used productively.

Smart Port Challenge (20): A public event as a method to innovate and to generate new ideas in the context of maritime logistics. The event has been hosted at least once.

River Navigation (21): Sensor and simulation project to increase accuracy of under-keel depth estimation, thus allowing more efficient planning of port basin and river utilization. This solution is used productively.

Remote-controlled Infrastructure (22): Automation project employing sensors and actors to allow remote control of movable infrastructures such as locks and bridges. The solution is still in pilot state.

Barge Traffic System (23): Process digitization for terminal slot management, allowing more efficient and less reactive traffic coordination. This solution is in full productive usage.

Gas Detectors (24): Distributed mesh of gas sensors aimed at noticing gas leaks in real-time at dozens of locations throughout the port area. The solution is used productively.

\section{Methodology}

The conducted research follows the principles of Action Design Research (ADR) as formalized by Sein et al. [13]. In accordance with ADR, the research project was initiated by the Hamburg Port Authority's (the practitioner's) problem to gather and systematically document information about smart solutions developed and/or implemented at other ports (principle 1: practice-inspired research). While creating a formalized descriptive model of IT solutions, the focus of the research project was to develop an information system incorporating the model as well as shaping a new communication channel to communicate the documented solutions. Therefore, we classified our research to follow an IT-dominant Building, Intervention and Evaluation (BIE) task.

The research team was composed of a senior researcher and a $\mathrm{PhD}$ student, and our area of interest was how our emerging definition of the term solution [10] could communicated in a more formalized matter and how the portrayal would shape the communication, an essential part in the diffusion of innovation [9] (principle 2: theory-ingrained artifact). To align artifact shaping and organizational context, regular meetings every two to three weeks were implemented with a subset of the participating 
practitioners (principle 3: reciprocal shaping). During these meetings, the researchers' theoretical background was used to progress into being able to answer the practitioners' questions, while the practitioners' insights and knowledge were leveraged to ensure the usability of the artifact, i.e. its effectivity, its efficiency, and the perceived satisfaction during application [14] (principle 4: mutually influential roles).

Although this ensured a continuous alignment with a very small group of practitioners, we did several evaluation cycles and with each cycle we increased the number of participants as well as the organizational scope of their roles (principle 5: authentic and concurrent evaluation). The first evaluation cycle with participants other than those attending our regular meetings was with a subject expert (head of department). The second evaluation took place with anticipated end-users at the port of Hamburg. To broaden the organizational scope beyond the port of Hamburg, the third evaluation cycle was conducted with an anticipated later user at another European port participating in the chainPORT initiative, and our fourth and last evaluation cycle was done in a group meeting with chainPORT members of multiple continents.

The continuous development of the ensemble artifact was guided by principles on several levels. Most importantly, we followed an concern-based development of the underlying architectural meta-model. These architectural concerns ensured an alignment of theoretical background and organizational context (principle 6: guided emergence). The stakeholders of these concerns were identified by a stakeholder analysis guided by [15], using semi-structured interviews to identify stakeholders (step 1). We then used reconstructive, bottom-up categorization of these stakeholders by employing a stakeholder-led categorization approach (step 2). To grasp the responsibilities, capabilities and social interplay of the stakeholders, a mixed approach was taken to investigate the relationships between stakeholders (step 3).

To generalize our findings, we conceptualize our IS-based approach to the specific challenges encountered in our research project's setting (principle 7: generalized outcomes). Accordingly, we focus on how the solution to the research problem was found and which conceptual problems needed solving, allowing our approach and/or learning to be leveraged in similar settings.

\section{Related Literature}

Several streams of scientific research were found to be relevant for our research project. We looked at Internet of Things literature to better understand the IT solutions in place at the ports, as most of those projects were IoT projects or very similar to IoT projects. To document solutions in a uniform way, enterprise architecture management was expected to be a good foundation, as its core concept is to link different aspects of projects / solutions / IS artifacts with each other to create a high-level understanding of the subject matter. The process of sharing the gathered knowledge and all related aspects are described and formalized in the rich body of innovation diffusion research.

\subsection{Internet of Things}

The term Internet of Things is less a well defined term but rather an umbrella term [16] for many different aspects of technological systems and physical objects, and their relationships and interconnectedness, and the application of IoT is seen in many different types of industries [17]. An important perspective is to acknowledge three different aspects of the subject, an internet-oriented vision, a things-oriented vision and a semantics-oriented vision [18], defining the IoT paradigm as the intersection between them. These perspectives were applied to the port authority context in [11] by choosing different levels of detail or abstraction for different kinds of objects, focusing either on the thing-aspect of IT or on connectivity-related aspects to tackle the overarching problem of documenting semantic relationships within IT architectures.

\subsection{Enterprise Architecture Management}

The main goal in enterprise architecture management is to create a meaningful link between different, specialized architectures [19]. In practice, EAM can serve both as a planning tool and as a representation of an enterprise's current state [20], and has matured over the last decades to be a well-researched field [21].

The link between IoT and EAM has been drawn in the past, with a focus on technology-related architectural layers $[11,8]$. This research builds upon the smart brick concept introduced by Schirmer et al. [11], which addresses the thing-aspect of the Internet of Things. Smart bricks are an abstraction from sensors and are virtual entities combining a physical object with attached sensors. Thus, sensors are described by their role when attached to an object, e.g. an induction loop (sensor) would instead be described as 
a car-counting road segment (combination of sensor and physical object).

Drews et al. [8] use architectural slices to describe IT solutions, a viewpoint that is related to depicting projects. Still, a solution may be changed, adapted or refactored throughout several projects, so a distinction between solution and project should be made from an architectural perspective. From an architectural perspective, descriptions of solution architectures are either only valid in a very specific context [22] or the description is very abstract [23]. An outline of what constitutes an IT solution in the context of maritime logistics has been made [10], and research indicates that the character of an innovative IT solution spans beyond the essential architectural layers outlined by Winter et al. [19].

\subsection{Innovation Diffusion}

The diffusion of innovation is the process in which an innovation spreads through a group of organizations [1]. This field has a rich body of scientific research and guided our research by providing an encompassing process model with a detailed framework of descriptions of relevant artifacts. Most importantly, Rogers [9] stresses the close relationship to communication and the impact that communication channels have. $\mathrm{He}$ also breaks the diffusion process down to (1) gaining knowledge of an innovation, (2) persuasion through perceived characteristics, (3) decision to adopt or reject, (4) implementation and (5) confirmation. The assimilation of an innovation is defined as the respective process within an organization that adopts an innovation, which follow the same steps from gaining knowledge (1) to implementation and confirmation (5), but from a single organization's perspective.

The goal of our research project was to assist in the assimilation process, i.e. to help organizations within the stated process. Since most of the regarded innovations were relatively new to the maritime logistics industry and the practitioner's stated goal was to increase effectiveness and efficiency in cross-organizationally assimilating innovations, a desire to be placed in the early adopter bracket (as defined by Rogers [9]) is implied. Therefore, several attributes of early adopters can be assumed to describe the practitioners, most noteworthy their greater ability to deal with abstractions and uncertainty, greater rationality and a positive bias towards change [9]. Therefore, we identified the need to abstract from details, even though the reduction of details increases uncertainty in certain aspects. This connects well to the concepts of enterprise architecture management, which also tries to neglect details while describing the high-level relationships between architectural artifacts.

During our research, we focused on the first three stages of the process, while keeping in mind that later stages may possibly be supported in yet to be planned research projects. Regarding the knowledge phase (1), Rogers [9] identified socioeconomic characteristics, personality variables and communication behavior as important factors. Our IS-based approach focuses on formalizing and improving the communication behavior by providing a technology-based communication channel for innovations and reducing the need of informal communication, thus reducing the overhead in (1) gaining knowledge of an innovation.

The (2) persuasion phase identified by Rogers depends most notably on the perceived characteristics of an innovation, namely its relative advantage, compatibility, complexity, trialability and observability [9]. Throughout our research, we aimed to solve these specific problems by building upon the architectural description of IT solutions by Tesse et al. [10], and formalizing visualization, data collection, specific attributes and relationships by means of a web-based software that was developed during our research project.

\section{Results}

We created a piece of software that allowed us to iteratively develop a suitable meta model to architecturally describe the IT solutions investigated within the IT solutions workgroup of the chainPORT network. We will first describe the derived model as well as viewpoints / visualizations, and secondly attempt to generalize our findings by describing how the chosen development approach and data model was beneficial and how it might help research projects in other organizational settings.

\subsection{Map View / Global View}

Enterprise architecture tools commonly focus on architectural components displayed in table-like forms or in different diagram types. This makes sense as enterprise architecture management typically abstracts from object instances and only regards object classes and their relationships. While the possible need to regard object instances has been mentioned [11], we encountered several architectural concerns where instance knowledge was necessary. To cope with the increasing numbers of objects at display, we needed to find suitable a viewpoint on our collected data. Systems that solve this problems are for example configuration management databases (e.g. as proposed 
by ITIL ${ }^{\circledR}$ [24]) or conceptually related geo information systems. Especially geo information systems usually depict objects on a map. We adopted this approach and created a map-based viewpoint on architectural data. The goal of this viewpoint is to depict information contextually, i.e. allowing users to understand the purpose of objects by seeing how they are situated. This is necessary, as anticipated users have a large variety of educational background, ranging from topic experts to IT specialists and managers. And example are locks, as they can solve different purposes which are easily understandable when displayed on a map, whereas the knowledge of what characterizes a "flash lock" cannot be assumed for all users.

Because of the amount of objects to display on a physical map, this viewpoint is not automatically useful. Instead, the complexity was reduced by introducing a filter concept and by binding levels of detail to zoom-levels on the map. Starting with a world map, one can see all the participating ports of the chainPORT initiative. In this viewpoint, a filtering mechanism can be used to highlight ports that match certain criteria as defined in our collected architectural concerns. These include for example highlighting ports having certain types of physical objects, for example movable bridges. Another filter was to highlight ports that have piloted or productive IT solutions concerning the selected physical objects. Filtering on other solution attributes, for example based on the usage of certain innovational technologies or paradigms, was deemed necessary as per the collected architectural concerns, but was not implemented in the evaluated prototype.

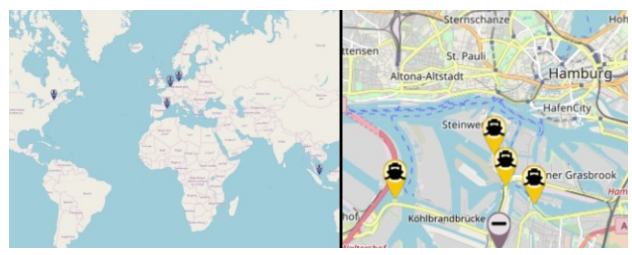

Figure 1. Screenshots showing a low level of detail when zoomed out (left side) and a high level of detail when zoomed in (right side).

Source: Map material (C)OpenStreetMap [25]

When zooming into a specific harbor, the level of detail would switch, depicting actual physical objects within the harbor limits, as depicted in figure 1. Furthermore, the detailed display of a port was further influenced by the applied filters, i.e. highlighting physical objects that are linked to solutions or that are a sub-type of the filtered object class.

\subsection{Solution Details}

The solution details were modelled leveraging the modelling techniques of previous research $[11,8]$. As an

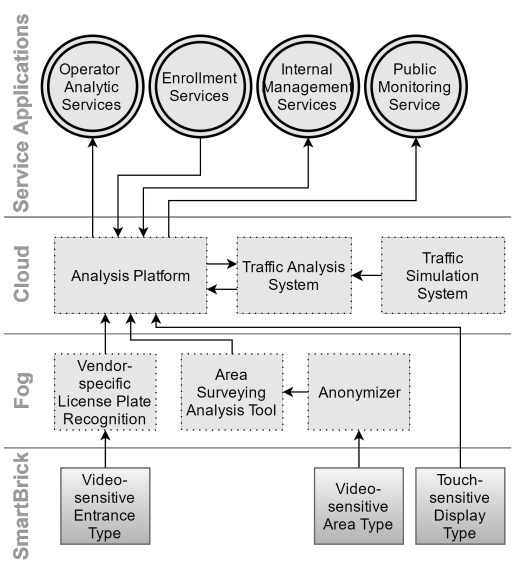

Figure 2. Technology layers of a sample solution architecture, modelled following [11].

example of this modelling paradigm, figure 2 shows the PrePORT Parking project's solution architecture with the solution's main information system, an analysis platform, at the center. This solution addresses the problem of scarce parking lot availability within the port limits and is piloted at a parking lot outside the main port area. The main issue for the trucker is that they need to be at a specific terminal at a certain time, but parking outside of the port area introduces a risk of not reaching the terminal in time as the road traffic is hard to predict. The solution tackles this problem by leveraging another solution's traffic forecast data, which is generated by complex simulations on data from various sources, including in-road induction loops, video cameras and passive wireless device id recognition. As the traffic simulation system is part of another solution, these sensor types are not displayed in the PrePORT Parking solution's architecture. This information would be available in the respective solution's architecture.

The displayed operator analytic services target reporting and internal utilization monitoring, as indicated in figure 3. As the analysis platform tries to create lanes of vehicles wanting to leave at the same time, the enrollment service asks truckers for their destination and desired time of arrival. This information is then combined with detailed traffic forecasts from the traffic simulation system to estimate a resonable departure time for each truck. Then, the trucker is shown individual instructions on large boards at the parking lot (vehicle recognition by license plate), alongside with general parking lot utilization information (public monitoring services). 


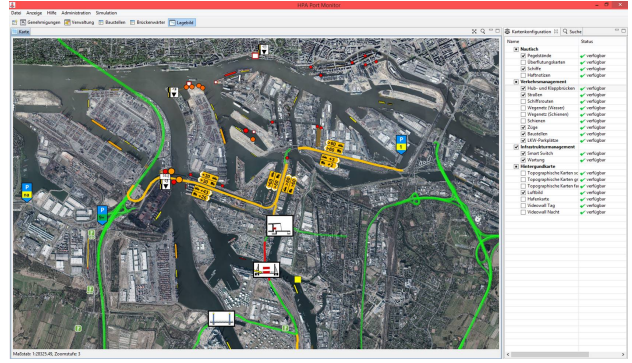

Figure 3. Monitoring system for port-wide traffic data.

Source: Screenshot provided by the Hamburg Port Authority, AR

These solution details target step (2) of the assimilation process and display the perceived characteristics of solutions. The technological solution architecture specifically targets the ability to gauge a solution's compatibility and complexity. The other attributes stated by Rogers [9] are dealt with by documenting solution attributes identified by Tesse et al. [10], namely a solution description, a solution's relative advantage and a description of the problem that the solution attempts to solve, thus allowing other organizations to trial the solution.

\subsection{Comparison View}

To align the derived use cases and specific stakeholder needs with the IS, an in-between viewpoint needed to be introduced, with the purpose of comparing architectural entities, as seen in figure 4. Firstly, this screen is necessary to compare organizations from a high-level perspective, to see whether or not they are comparable. This information includes statistical data of the organization and its documented physical objects, in the context of ports for example the container throughput, degree of containerization, average bridge length or average age of a port's ship locks. Therefore, a necessity exists to make organizations comparable, which is most probably highly dependant on the industry and focuses on aspects that vary among organizations of the reviewed type and within the documented group of organizations.

\subsection{Types of Relationships}

Throughout our research we found four kinds of relationships: linked to, part of, extends and implements relationships. Part-of relationships are very common to any system documenting physical entities. The part-of relationship-type allows for a very natural way of hiding details and reducing complexity, and

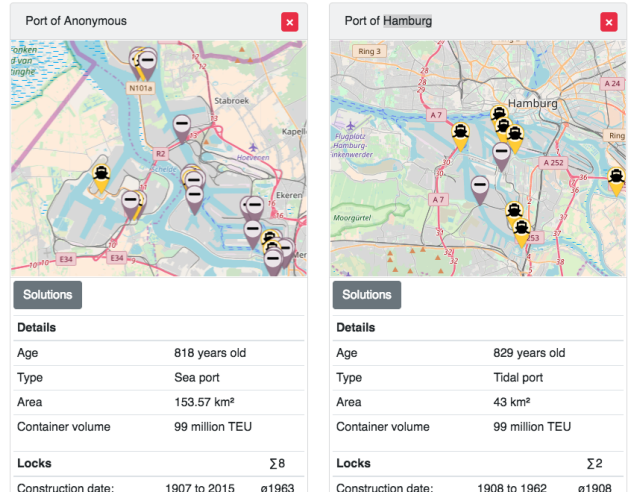

Figure 4. Screenshot showing the comparison view of two ports

Source: Map material (C)OpenStreetMap [25]

accordingly proved very useful when documenting hierarchies of physical objects. Furthermore, this allows for more comprehensive ways of filtering data, which implies that the concept of part-of relationships might prove useful elsewhere, for example when embedding a solutions processes into an overarching enterprise process architecture.

While common in software engineering, the extends and implements relationships seemed useful in combination with our specific data model. Since the created software has an adaptable meta model that can be changed at runtime, the implements relationship indicates that a certain architectural entity is described by a certain meta model class. Furthermore, the extends relationship is a special case of the implements relationship and describes the a meta model class is described by another meta model class and further describes it by adding more attributes. As an example, both movable bridges and locks can be specified as extending the movable infrastructure class, and the attribute "vehicles passing per year" is stipulated by the movable infrastructure class. Instances exist, where extended objects are directly instantiated, but we found those to occur solely when sufficient documentation to further specify an object was not present.

\subsection{Role-based Enterprise Architecture}

To allow for faster development of the organizational aspects of our IS, a role-based data model was chosen. This differs from instantiation in that an a class instance has exactly one class-template, whereas our role-based model allows objects to take on multiple roles. We found this approach to allow slightly more flexibility at early modeling stages, but the current state of documentation does not have a single object that does not take on exactly one role. Therefore, we find the concept of 
role-based EAs slightly beneficial for developing new modelling concepts, but do not recommend productive usage in matured architecture tools, as there appears to be no evidence that a matured model needs this kind of flexibility.

\subsection{Types of Attributes}

Since the created IS allows changes to the meta model at runtime, special care needed to be tended to the attribute types. We identified the types text, number, position, enumeration, link and map of numbers. We needed to introduce the position attribute-type to allow documenting physical objects. With our model of class inheritance, a sub-class of a class with a position would inherit a position attribute. We did not find any situations where this behavior was not semantically correct and anticipate that the position attribute-type is a base type. To allow for varying levels of details, it is (in our software) defined by 4 values: latitude and longitude are set on a per instance level, the minimum and maximum zoom levels are set on a per class level.

The link attribute-type can model both logical relationships as well as part-of relationships. As our attributes are per class, links automatically have a cardinality of exactly one at its object instance of origin. Therefore, a link needs information of the destination cardinality, which is set on a per class level. We found that for class inheritance the cardinality would only decrease for sub-classes, i.e. an object of the subclass could link to less than or the same amount of object instances as the orignial class would allow. Common scenarios include reducing the cardinality from "any" to "one" or from "one" to "zero" (e.g. part-of relationships).

Enumerations seem to be a special case of text-based attributes, permitting only the selection of a specified set of attributes. We found this to be replaceable in many cases by creating sub-classes, but enumeration-type attributes allow faster and more comprehensible modeling, e.g. a movable bridge with hydraulic drives, rather than a sub-class "hydraulically movable bridge". In cases with multiple enumerations, sub-classes for any combination of the enumerations would have to be created. Therefore, we deemed enumeration-type attributes very useful in EA modelling.

Lastly, we identified map-like attribute-types for numbers, which were used for example to document the number of vehicles passing a bridge per year. Possible other use-cases include a more structured documentation of attributes per unit of interest, for example a responsibility per employee. In the special case of documenting amounts per year, we found it helpful to limit the available map keys to a specific set of years, so that the documentation process is faster and more uniform among objects in different organizations.

\subsection{Lessons Learned: Visualization}

Visualizations are strongly coupled with an architecture's meta model, and our meta model can be adapted at runtime. Hence, the visualizations would need to be adaptable at runtime too, to incorporate the changes done to the meta model. Because of the complexity innate to changes in the visualization, it is a difficult task to create a flexible visualization model editable at runtime that is not limited to very basic operations and is also able to create specific viewpoints. The approach taken to tackle this problem was to leverage the close relationship of our role-based approach with class inheritance models in software engineering, allowing us to create a class representation in our used programming language at runtime, which was then imported into the software's source code after meta model adaption cycles, which increased the development speed at the cost of an conceptual gap within the software and meta model development process. This approach caused less issues than anticipated, and the conducted rapid feedback cycles when developing the architectural meta model proved to create a stable core structure very early on, and further improvements mostly refined the used attributes and created new specialized types inheriting from stable core classes.

\subsection{User Feedback}

The general feedback during our artifact's evaluations was positive, and we can confirm that the approach of leveraging both innovation diffusion research and EAM was seen to create added value for the interviewed practitioners. The formalization and modelling methods of EAM helped structuring and abstracting from the actually present ecosystem architecture, while innovation diffusion research provided a valuable framework for a guided overarching process. This was especially necessary for our inter-organizational environment of enterprises across the globe.

During the evaluation cycles one recurring concern was the non-existance of a data collection process, as EAM may fail to deliver value when data is incomplete, not up-to-date or if commitment of key stakeholders is missing. Furthermore, documenting object instances instead of just classes of objects further increases the amount of time needed to document and to keep the data up-to-date. 


\section{Discussion, Limitations and Outlook}

Throughout the conducted research we found multiple occasions where more conceptual work was needed. In this section we list these occasions and give recommendations on how each problem can be addressed. Within each of the following subsections, there is one paragraph each for discussion, limitations and outlook.

\subsection{Gap: Flexibility in Modelling and Visualization}

The indicated gap in flexibility between modelling and visualization (see 5.7) stems from editing each one of them in a different conceptual layer of the application: The model is edited at run-time while visualizations were created in code. This offered great flexibility for both aspects - for modelling by allowing rapid model prototyping during interviews, as allowed by quick and easy meta model changes, and for creating targeted visualizations, as we were not limited to a predefined set of available visualizations.

Both the convenience in changing the meta model and the flexibility in visualizing significantly slowed down the generation of visualizations. This was observed to negatively impact the speed of feedback cycles for the application, as discussions tended to stay theoretical while concepts evolved at a faster pace than their visualization.

We found no situations contradicting with class inheritance models of scripting languages that would prevent the mapping of our role-based approach to a class-based model. We would very much like to see how modelling best practices in programming languages might benefit agile meta model prototyping in enterprise architecture management.

\subsection{Concept for Data Collection}

Enterprise architecture management is seen to incorporate more and more data in order to answer the posed concerns. This needs to be an anticipated problem, and was somewhat anticipated in our research project but not formalized. After the research project is finished, the port authorities need to be able to manage their data to keep it up-to-date.

We did enter data as the tool evolved, but notice that levels of granularity and data quality (especially age) vary among our documented solutions. This diminishes the trust in the tool itself, as participants cannot easily gauge the quality of the data they look at.

We propose further research on automating the process for some of the architectural layers. This automization can be done by either connecting to specialized tools (automated data collection) or by incorporating some of the specialized architectures into EAM (convergent paradigms). To achieve this, further generalization on types needs to be done and a more complete, stable and detailed meta model needs to be in place, e.g. by defining common components within specific architectural layers [26].

\subsection{Shareability of Solutions}

The emerged ensemble artifact is targeted at supporting the cross-organizational assimilation of solutions, and the created software does address the needs of the assimilation process, as formalized in innovation management theory.

The concepts need to be tested on a more complete dataset of the chainPORT participant's respective architectures, as only some projects have been modelled and the majority of the modelled solutions are still missing some specific details. We have established new ways of gaining knowledge of innovations, but a holistic view on an integrated architectural landscape is yet missing. Additionally, no solution has yet been fully assimilated by another port authority, so only the first steps of the innovation adoption process formalized by Rogers (knowledge, persuasion) [9] have been supported.

We anticipate to extend our research to accompanying the actual assimilation of a port's innovation by another port. The learnings from accompanying the adoption / assimilation of one solution by another port may refine and extend the model, allowing it to aid at later stages of Rogers' innovation diffusion process, namely step (3) decision to adopt or reject and (4) implementation [9]. To guide the decision-making process, an IT artifact can provide communication methods such as ratings and comments per stakeholder, which would yield detailed knowledge about how decisions are made. Interesting parameters are the number of stakeholder involved in a decision, seeing which steps of the decision-making process run in parallel and which run sequential, and lastly by outlining communication patterns and behavior and if it is correlated to other factors.

\subsection{Towards a Core Enterprise Architecture}

The concept of a core EA [10] in contrast to a solution EA may benefit from further research. Solutions are always embedded into an enterprise and have links to several architectural entities that cannot be attributed to any specific solution, and the entities that a solution is embedded to can be characterized as 
the core architecture. We found that some stakeholder concerns can only be answered by looking at the port's core EA rather than a solution EA, as it holds detailed knowledge about how solutions are embedded into an organization and at what levels an organization employs standardization.

Currently, there is scarce knowledge of this topic and its implication on the diffusion of innovation, i.e. whether certain types of core EAs promote modularity and thus benefits an organization's ability to adopt or share innovations. We anticipate that a core architecture is strongly related to the researched industry's context, in our case the maritime logistics industry with a specific focus on port authorities.

A core enterprise architecture may further promote the ability to assimilate IoT innovations and we look forward to explore this subject in the chainPORT initiative's inter-organizational setting. A possible next step could be the identification of a core architecture's scope and attributes important to innovation diffusion, which may later lead to partial standardization or to enabling strategic development of an enterprise's core architecture.

\section{References}

[1] R. G. Fichman, "The diffusion and assimilation of information technology innovations," Framing the domains of IT management: Projecting the future through the past, vol. 105127, 2000.

[2] Gartner, "Five megatrends shift the computing landscape," Gartners hype cycles for 2015, 2015.

[3] F. Wortmann and K. Flüchter, "Internet of things," Business \& Information Systems Engineering, vol. 57, no. 3, pp. 221-224, 2015.

[4] E. Abad, F. Palacio, M. Nuin, A. G. De Zarate, A. Juarros, J. Gómez, and S. Marco, "Rfid smart tag for traceability and cold chain monitoring of foods: Demonstration in an intercontinental fresh fish logistic chain," Journal of food engineering, vol. 93, no. 4, pp. 394-399, 2009.

[5] M. Fruth and F. Teuteberg, "Digitization in maritime logisticswhat is there and what is missing?," Cogent Business \& Management, vol. 4, no. 1, p. 1411066, 2017.

[6] B. Montreuil, "Toward a Physical Internet: meeting the global logistics sustainability grand challenge," Logistics Research, vol. 3, no. 2-3, pp. 71-87, 2011.

[7] B. Wiegmans and S. Dekker, "Benchmarking deep-sea port performance in the Hamburg-Le Havre range," Benchmarking: An International Journal, vol. 23, no. 1, pp. 96-112, 2016.

[8] P. Drews, I. Schirmer, J. Tesse, S. Saxe, and U. Baldauf, "Internet of Things-specific Challenges for Enterprise Architectures Internet of Things-Specific Challenges for Enterprise Architectures: A Cross-Case Comparison of Explorative Projects from the smartPORT Initiative," Twenty-third Americas Conference on Information Systems, 2017.
[9] E. M. Rogers, Diffusion of Innovations. Free Press, 5th ed., 2003.

[10] J. Tesse, I. Schirmer, P. Drews, S. Saxe, and U. Baldauf, "Supporting diffusion of iot solutions exemplified by the chainport initiative," 2018.

[11] I. Schirmer, P. Drews, S. Saxe, U. Baldauf, and J. Tesse, Extending enterprise architectures for adopting the internet of things Lessons learned from the smartPORT projects in Hamburg, vol. 255. 2016.

[12] Hamburg Port Authority, "Press release: Foundation of the global port network ChainPORT," 2018. https: / / www. hafen-hamburg.de/en/news/---34590 (accessed 2018-06-14).

[13] M. K. Sein, O. Henfridsson, S. Purao, M. Rossi, and R. Lindgren, "Action design research," MIS quarterly, pp. 37-56, 2011.

[14] E. Frøkjær, M. Hertzum, and K. Hornbæk, "Measuring usability: are effectiveness, efficiency, and satisfaction really correlated?," in Proceedings of the SIGCHI conference on Human Factors in Computing Systems, pp. 345-352, ACM, 2000.

[15] M. S. Reed, A. Graves, N. Dandy, H. Posthumus, K. Hubacek, J. Morris, C. Prell, C. H. Quinn, and L. C. Stringer, "Who's in and why? A typology of stakeholder analysis methods for natural resource management," Journal of environmental management, vol. 90, no. 5, pp. 1933-1949, 2009.

[16] A. Bassi, M. Bauer, M. Fiedler, T. Kramp, R. Van Kranenburg, S. Lange, and S. Meissner, "Enabling things to talk," Designing IoT Solutions With the IoT Architectural Reference Model, pp. 163-211, 2013.

[17] E. Borgia, "The internet of things vision: Key features, applications and open issues," Computer Communications, vol. 54, pp. 1-31, 2014.

[18] L. Atzori, A. Iera, and G. Morabito, "The internet of things: A survey," Computer networks, vol. 54, no. 15, pp. 2787-2805, 2010.

[19] R. Winter and R. Fischer, "Essential layers, artifacts, and dependencies of enterprise architecture," in Enterprise Distributed Object Computing Conference Workshops, 2006. EDOCW'06. 10th IEEE International, pp. 30-30, IEEE, 2006.

[20] L. Kappelman, T. McGinnis, A. Pettite, and A. Sidorova, "Enterprise architecture: Charting the territory for academic research," AMCIS 2008 Proceedings, p. 162, 2008.

[21] D. Simon, K. Fischbach, and D. Schoder, "An exploration of enterprise architecture research.," CAIS, vol. 32, p. 1, 2013.

[22] J. Banerjee and S. Aziz, "SOA: the missing link between enterprise architecture and solution architecture," SETLabs briefing, vol. 5, no. 2, pp. 69-80, 2007.

[23] The Open Group, "The open group architecture framework," 2018. http: / / www.opengroup. org/togaf / (accessed 2018-06-14).

[24] AXELOS limited, "IT Infrastructure Library ${ }^{\circledR}$, 2018. https://www.axelos.com/ (accessed 2018-06-14).

[25] Open Street Map, “openstreetmap.”

[26] A. Josey, "The open group it4it reference architecture, version 2.0," Berkshire, UK: The Open Group, 2015. 